\title{
Positive and Negative Feedback in Engineering and Biology
}

\author{
E. S. Zeron ${ }^{1}$ \\ Depto. de Matemáticas, CINVESTAV, Apartado Postal 14-740 \\ 07000 Méxido D.F., México.
}

\begin{abstract}
No other concepts have shaken so deeply the bases of engineering like those of positive and negative feedback. They have played a most prominent role in engineering since the beginning of the previous century. The birth certificate of positive feedback can be traced back to a pair of patents by Edwin H. Armstrong in 1914 and 1922, whereas that of negative feedback is already lost in time. We present in this paper a short review on the feedback's origins in the fields of engineering and biology. Besides, we compare the main feedback's ideas in control theory and system biology in order to get a better understanding of the lactose and tryptophan operons' regulatory systems (control systems). It is obvious that we need to know the genome of an organism in order to understand how it works, but that is only half of the puzzle, for we also need to know how the underlying genetic regulatory systems work in order to get a complete picture of the cell's dynamics.
\end{abstract}

Key words: positive and negative feedback, gene networks, tryptophan operon, lactose operon, control theory, regenerative and super-regenerative circuits

AMS subject classification: 92B05, 93A30

\section{Introduction}

Like with many ideas in science, the concept of feedback appeared in several branches of the human knowledge at almost the same time; but generally, with very different histories, evolutions and consequences. We present in this paper a short review on the origins of positive and negative feedback in the fields of engineering and biology. Besides, since feedback has been one of the main

\footnotetext{
${ }^{1}$ Email: eszeron@math.cinvestav.mx
} 
tools in engineering for the last sixty years, in and after the World War II, we also compare the main feedback's ideas in control theory and system biology in order to get a better understanding of the lactose and tryptophan operons' control systems (control loops).

\subsection{Engineering antecedents}

No other concepts have shaken so deeply the bases of engineering like those of positive and negative feedback. They have played a most prominent role in engineering since the beginning of the previous century. We can find them almost everywhere; actually, it is really difficult to find an appliance not involving some kind of feedback. Nevertheless, these two concepts arouse at very different places and dates. The birth certificates of positive feedback can be traced back to a pair of patents by Edwin H. Armstrong. He patented the regenerative circuit in 1914 [3] and the super-regenerative circuit in $1922[4,5]$. Besides, there are some strong evidences that Nikola Tesla worked on the regenerative circuits back in August 1899 [25]; but he did not continue with his research because the first practical electronic amplifier (the triode) was not invented until 1907.

It did not take longer before the radio community discovered two of the main implications of the positive feedback: oscillations and bistability; the Great War only delayed these discoveries. Specialised oscillation circuits were invented by Hartley in 1915 [19] (patented until 1920), by Colpitts in 1919 [12, 11] and by Turner in 1920 [40, 41]. The paternity of the bistable circuits (flip-flop and multivibrators) is mainly disputed by two teams [13]: a French one composed by Abraham and Bloch who presented their results in June 1919 [1, 2], and a British one composed by Eccles and Jordan who presented their results in September 1919 [14, 15, 16]. Moreover, the now universal Schmitt trigger was invented nineteen years later, in 1938 [37].

On the other hand, the birth certificate of negative feedback is already lost in time. One of the first artificial automatic regulatory (negative feedback) systems, a water clock, was invented by the technician Ktesibios (285-222 BC) in the Hellenistic Greece. In that clock, water flowed from a source - such as a holding tank - into a reservoir, then from the reservoir to the mechanisms of the clock. Ktesibios's device used a cone shaped float in order to monitor the level of the water in the reservoir, and to maintain this level constant by adjusting the water flow. This simple mechanism assured that the reservoir never overflowed or ran dry. This is the first artificial truly automatic selfregulatory device that required no outside intervention between the feedback and the controls of the mechanism. Several centuries later, two of the main forces behind the first industrial revolution were the steam engine and the centrifugal governors used as a control system for keeping the steam engines running at a constant speed. In 1868, James Clerk Maxwell published a theoretical article on governors [26], being one of the first to discuss and refine the principals of self-regulating devices.

After Maxwell's paper, the ideas on negative feedback sparked a revolution in engineering and initiated new branches of science like cybernetics and control theory. We review below some of the seminal works on cybernetic. In 1935, the Russian physiologist P. K. Anokhin presented the lecture Unity of the Center and Periphery in which the concept of feedback (reciprocal afferentation) was studied [38, p. 270]. The Romanian scientist Ştefan Odobleja published Psychologie consonantiste in 1938 [30], describing many cybernetic principles. The study and mathematical modelling of 
regulatory processes became a continuing research effort and two key articles were published in 1943. These papers were Behaviour, Purpose and Teleology [32] by Arturo Rosenblueth, Norbert Wiener and Julian Bigelow; and A Logical Calculus of the Ideas Immanent in Nervous Activity [27] by Warren McCulloch and Walter Pitts. Finally, the classical text on cybernetics was written down by Wiener in Paris (1948) Cybernetics, or Control and Communication in the Animal and Machine [42].

It is quite interesting to notice that almost all the authors of the articles and books reviewed in the previous paragraph were working on biology or related fields: Anokhin worked on physiology, Odobleja on psychology, Rosenblueth and McCulloch on neurophysiology, and Pitts on cognitive psychology.

The papers by Maxwell (On governors) in 1868 [26] and Minorski (Directional stability of automatic steered bodies) in 1922 [28] are considered to be the birth certificates of control theory, for they made the first complete mathematical analysis of the negative feedback loops, and they introduced the concept of (the now universal) Proportional-Integral-Derivative control (PID control) as well. Posterior applications of the PID control to time delayed systems were analysed by Callender, Hartree, Porter and Stevenson in 1936 and 1937 [7, 20]. Finally, the first analysis on the effects of negative feedback in an electronic amplifier was done by Black in 1932 [6]; and some of the first applications and analysis of general servomechanisms can be traced back to Hazen [21] and Ivanoff [22] in 1934.

\subsection{Some biological antecedents}

Speaking about the feedback origins in Biology is not easy because nature has been using feedback loops for millions of years, and because biologists in general discovered the principles of feedback centuries ago. The concept of negative feedback keeps popping out into our faces day after day. Properly speaking, it literally keeps popping out into our eyes, for the pupils light reflex in our eyes is the perfect example of a negative feedback loop. When light levels are high, the pupil contracts, reducing the light flux onto the retina. The size of the pupil is controlled by circularly arranged constricting muscles, which are activated and inhibited (left to relax) by control signals from the brain. More light causes activation of the constricting muscles, which shrinks the pupil area and limits the light flux at the retina.

The pupil light reflex is a particularly attractive example of physiological feedback. Firstly, the state variable, the retinal light flux, is an intuitive quantity, and the actuator mechanism, pupil size, is easy to understand. Secondly, one can break open the feedback loop using a simple trick. Ordinarily, the incident light flux covers an area larger than the pupil, so that adjustments to the pupil size adjust the retinal light flux. If, however, one uses a beam of light that is narrower than the minimum pupil size, adjusting the area will not change the retinal light flux. The feedback loop is broken open, and one can then study how variations in the light intensity change the pupil size. For example, while we do not have space to describe the exact pupil reaction, its most important feature is that the pupil response to a changing light stimulus is delayed by about $300 \mathrm{~ms}$ [23]. This leads to an amusing consequence. If a narrow beam of light shines on the edge of the pupil, the pupil will begin to contract. Because of the delay, it will continue to contract after it is small 
enough that the beam no longer enters the pupil. After the delay, the eye realises there is no light and starts to enlarge the pupil. This continues, because of the delay, somewhat past the moment when the pupil is large enough to admit light to the retina. The system then begins to contract, and thus continues on, in steady oscillation [23].

Maybe the first physiologist to study negative feedback systems was Walter N. Cannon, who coined the term homeostasis in 1932 [8]. Moreover, as we have already said, some of the first analysis on negative feedback systems (and cybernetics) were done by psychologists and physiologists like: Anokhin in 1935 [38]; Odobleja in 1938 [30]; Rosenblueth, Wiener, and Bigelow in 1943 [32]; and McCulloch and Pitts in 1943 [27]. More recently, as biology has acquired the molecular tools that allow mechanistic insights into the cellular processes, it has become clear that the principles of feedback (both positive and negative) are indeed largely used in almost all the cell signalling and regulatory pathways. Negative feedback occurs when, for example, a signal induces the expression of its own inhibitor; and it serves to dampen or limit signalling. Positive feedback occurs when a signal induces more of itself, or of another molecule that amplifies the initial signal, and this serves to stabilise, amplify or prolong signalling. The widespread use of feedback and the variety of its consequences make it an important principle in cellular biology.

Freeman published in 2000 [17] a very nice recollection of biological regulatory systems where the positive and negative feedback cycles are used for temporal and spatial control of signalling (in order to limit or prolong the duration of a signal), for inducing pattern formation (establishing left-right asymmetry) via multiple integrated feedback cycles, et cetera. Furthermore, negative and positive feedback are well known for their capability of respectively inducing stability and multistability. The inducible lactose (lac) operon in E. coli is the classic example of bistability. It was first noted by Monod and coworkers more than fifty years ago, although it was not fully recognised at the time. The bistable behaviour of the lactose operon has been the subject of a number of studies. It was first examined in detail by Novick and Wiener in 1957 [29], and by Cohn and Horibata in 1959 [10]. Later, experimental studies include those of Maloney and Rotman in 1973 [24] and of Chung and Stephanopoulos in 1996 [9].

The next two sections of this paper are devoted to analysing the genetic regulatory systems of the tryptophan and lactose operons, which are two of the most extensively studied molecular systems, and are the respective classical examples of negative and positive feedback regulatory loops in system biology. We shall analyse the tryptophan and lactose operons comparing their respective structures against two of the most widely used feedback systems in engineering: the proportional-integral control and the regenerative circuit. These feedback systems have been very useful in engineering since the first industrial revolution; and we can expect that the feedback concepts invented by engineers also appear in the genetic regulatory systems of different operons, for the engineering has the strange habit of imitating nature.

\section{Negative feedback, tryptophan operon}

Jacob and Monod originally introduced the concept of operon as a general theory for gene control. According to them, an operon is a set of structural genes preceded by a small DNA region 
where repression takes place and a mRNA polymerase (mRNAP) binds to initiate transcription. An operon is repressed when an active repressor molecule binds the DNA regulatory region blocking it and preventing the binding of a mRNA polymerase. Later, it was discovered that the regulation of genes is a much more complicated process. Indeed, it is not possible to talk of a general regulatory mechanism, as there are many, and they vary from operon to operon.

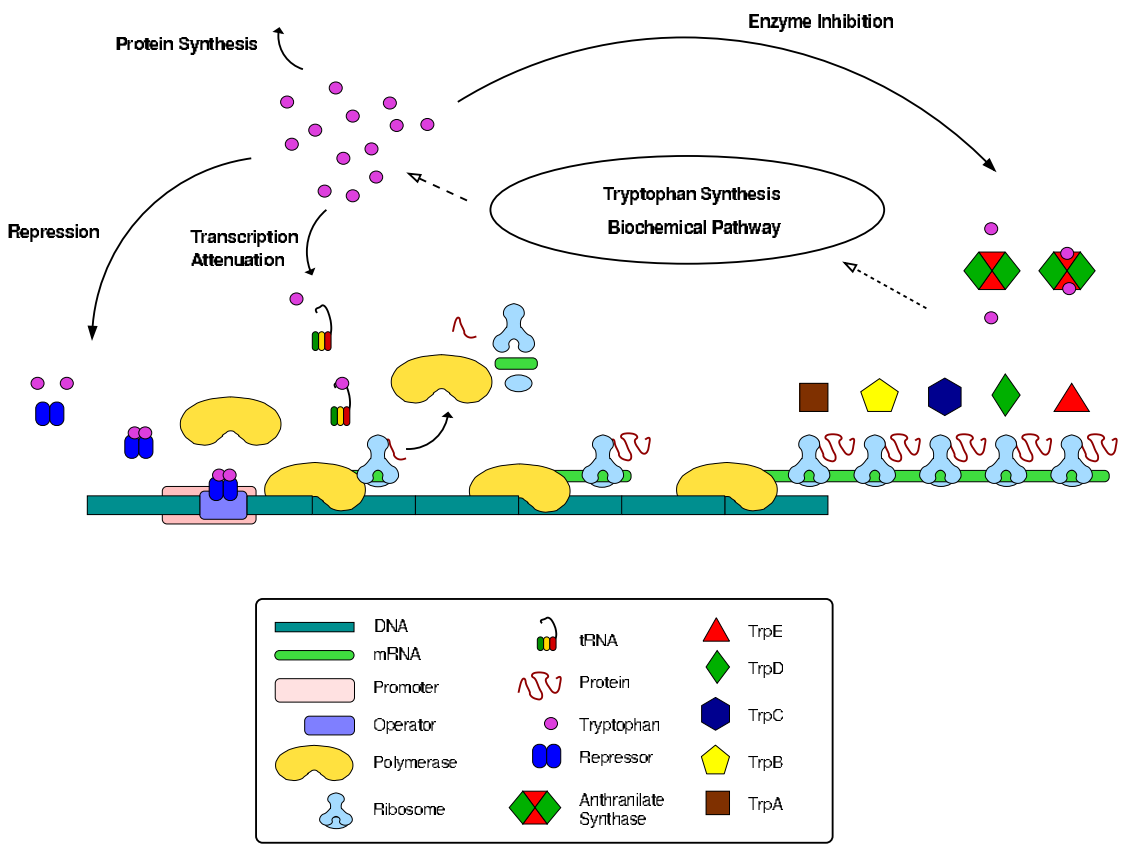

Figure 1: The tryptophan operon regulatory mechanisms. Figure adapted from Reference [35].

The synthesis of the amino acid tryptophan from chorismate is carried out by E. coli via a series of catalysed reactions. The catalysing enzymes are made up of the polypeptides encoded by the tryptophan operon genes. This operon consists of five structural genes, and it is also regulated by three different negative feedback mechanisms: repression, transcription attenuation, and enzyme inhibition. The action of the different feedback mechanisms can be seen in Figure 1: The repression loop works by preventing the binding of the polymerase to the DNA; the transcription attenuation loop works by promoting an early termination of the mRNA transcription; and the enzyme inhibition loop works by interfering in the enzymatic synthesis of the tryptophan from chorismate. The model equations derived in $[35,36]$ can be presented as follows:

$$
\begin{aligned}
\frac{d M}{d t} & =\mathcal{P}_{M}(T)-\left(\gamma_{M}+\mu\right) M, \\
\frac{d E}{d t} & =\frac{k_{E}}{2} M-\left(\gamma_{E}+\mu\right) E, \\
\frac{d T}{d t} & =\mathcal{P}_{T}(T) E-\rho_{0}(T) .
\end{aligned}
$$

Where $M, E$ and $T$ respectively denote the mRNA, total enzyme (anthranilate synthase), and total tryptophan intracellular concentrations. The functions $\mathcal{P}_{M}, \mathcal{P}_{T}$ and $\rho_{0}$ are all smooth, non- 
linear, and take only positive values. Moreover, $\mathcal{P}_{T}$ (resp. $\mathcal{P}_{M}$ ) is strictly-decreasing and represent the negative feedback mechanism: enzyme inhibition (resp. repression plus transcription attenuation). In the same way, $\rho_{0}$ is strictly-increasing and stands for the tryptophan consumption and diffusion rates inside the cell. Finally, we have the constants :

$$
k_{E} \approx \frac{30}{\min }, \quad \mu \approx \frac{0.01}{\min }, \quad \gamma_{M} \approx \frac{0.69}{\min }, \quad \gamma_{E} \approx \frac{0.0}{\min } .
$$

A complete description of the model $(2.1)-(2.3)$ can be found in $[35,36]$. In particular, this model describes a negative feedback mechanism because the functions which close the loops $\left(\mathcal{P}_{M}\right.$ and $\mathcal{P}_{T}$ ) are both strictly-decreasing. The bigger the concentration of tryptophan $T$ is, the smaller the values of the functions $\mathcal{P}_{M}(T)$ and $\mathcal{P}_{T}(T)$ are. We assert that the system (2.1)-(2.3) is actually the equivalent of a genetic Proportional-Integral (PI) control, where $T$ represent the variable to be controlled, the function $\mathcal{P}_{T}$ stands for the proportional part of the control, and the equations (2.1)-(2.2) stands for the integral part of the control. We present below an introduction to the Proportional-Integral control, in order to explain our results.

\subsection{The Proportional-Integral (PI) Control}

Consider the following one-dimensional differential model of a dynamical system, where $k_{0}>0$ is a given constant, $u$ is the entry variable, and $\rho_{0}$ is a smooth non-linear function,

$$
\frac{d x}{d t}=k_{0} u-\rho_{0}(x) .
$$

The main goal of control theory is to set the value of the entry variable $u$ in such a way that the exit variable $x$ is equal to a given reference $R$. The variable $x$ must follow the reference $R$, when it changes. The original theory for the Proportional-Integral control can be found in [26, 28], and the basic idea is to set the variable $u$ equal to a linear combination of the measured error $R-x$ and the integral of this error, that is :

$$
u:=C_{P}(R-x)+C_{I} \int(R-x) d t .
$$

Where $C_{P}, C_{I} \geq 0$ are the respective proportional and integral tuning parameters. By substituting the control (2.6) into our original dynamical system (2.5), we have,

$$
\begin{aligned}
\frac{d x}{d t} & =C_{P} k_{0}(R-x)+C_{I}^{1 / 2} k_{0} w-\rho_{0}(x), \\
\frac{d w}{d t} & =C_{I}^{1 / 2}(R-x) .
\end{aligned}
$$

The local stability of the system (2.7) around a given steady state $x^{*}$ can be easily determined by calculating whether the eigenvalues of the following matrix have both a negative real part,

$$
\left[\begin{array}{cc}
-C_{P} k_{0}-\rho_{0}^{\prime}\left(x^{*}\right) & C_{I}^{1 / 2} k_{0} \\
-C_{I}^{1 / 2} & 0
\end{array}\right] .
$$


It is easy to demonstrate that the proportional term $C_{P}(R-x)$ in (2.6) helps to strengthen the stability of (2.7). In particular, we have that (2.7) is strongly stable at $x^{*}$ whenever the control (2.6) is purely proportional; that is, whenever the proportional coefficient $C_{P} \gg 0$ is large enough and the integral coefficient $C_{I}=0$. Nevertheless, a purely proportional control is not a very good solution in terms of control theory, as the following analysis shows. We have to consider two different cases when we calculate the steady states of (2.7). If the control (2.6) is purely proportional $\left(C_{I}=0\right)$, the steady states of (2.7) are the solutions $x^{*}$ of the following equation,

$$
C_{P} k_{0}\left(R-x^{*}\right)=\rho_{0}\left(x^{*}\right) .
$$

Thus, it is almost impossible to set the steady state $x^{*}$ equal to the reference $R$, one of the basic demands in control theory, because this condition is only satisfy when $\rho_{0}\left(x^{*}\right)=0$. Moreover, it is also quite difficult to determine the exact value of the steady state $x^{*}$ as well, because the function $\rho_{0}$ in our original system (2.5) could only be an approximation, and the exact value of $x^{*}$ could be really sensible to small perturbations on $\rho_{0}$.

On the other hand, the steady state $x^{*}$ is obviously identical to the reference $R$, as we wanted, whenever the integral parameter $C_{I}$ is strictly positive in (2.7). Thus, we may conclude that the proportional parameter $C_{P}>0$ must be tuned to assure the stability of (2.7), while the integral parameter $C_{I}>0$ must be tuned to assure that the steady state $x^{*}$ is equal to the reference $R$.

\subsection{The tryptophan operon, a PI control}

Let us come back to analyse our tryptophan operon's control system (2.1)-(2.3). The differential equations can be rewritten as a one-dimensional dynamical system plus a negative feedback control loop :

$$
\frac{d T}{d t}=u-\rho_{0}(T) \text { plus } u:=\mathcal{P}_{T}(T) E,
$$

where $E$ is calculated from $\mathcal{P}_{M}(T)$ via :

$$
\frac{d^{2} E}{d t^{2}}+\left(\gamma_{M}+\gamma_{E}+2 \mu\right) \frac{d E}{d t}+\left(\gamma_{M}+\mu\right)\left(\gamma_{E}+\mu\right) E=\frac{k_{E}}{2} \mathcal{P}_{M}(t)
$$

Moreover, the functions $\rho_{0}, \mathcal{P}_{M}$ and $\mathcal{P}_{T}$ all smooth, non-linear and take only positive values. The steady state $T^{*}$ of the system (2.8)-(2.9) is the unique solution to the following equation (it has only one solution because the functions $\rho_{0}$ and $\mathcal{P}_{M} \mathcal{P}_{T}$ are respectively strictly-increasing and decreasing),

$$
\begin{aligned}
\rho_{0}\left(T^{*}\right) & =k_{0} \mathcal{P}_{M}\left(T^{*}\right) \mathcal{P}_{T}\left(T^{*}\right), \\
\text { with: } \quad k_{0} & :=\frac{k_{E}}{2\left(\gamma_{M}+\mu\right)\left(\gamma_{E}+\mu\right)} \approx 2.14 \times 10^{3} .
\end{aligned}
$$

We assert that the term $u=\mathcal{P}_{T}(T) E$ works as a Proportional-Integral (PI) control in the system (2.8); the factors $\mathcal{P}_{T}(T)$ and $E$ have the respective properties of a proportional and an integral control. We have already analysed in [36] the stability of the system (2.8)-(2.9) via the 
second Ljapunov's method. In particular, we showed in [36] that the factor $\mathcal{P}_{T}(T)$ in (2.8) strongly improves the stability of the whole system. For example, if we change $\mathcal{P}_{T}(T)$ in (2.8) by the constant term $\mathcal{P}_{T}\left(T^{*}\right)$, where $T^{*}$ is the steady state given in (2.10), and we run simulations of the system (2.8)-(2.9) using either $\mathcal{P}_{T}\left(T^{*}\right)$ or $\mathcal{P}_{T}(T)$, we shall see that the over-shots calculated with the constant term $\mathcal{P}_{T}\left(T^{*}\right)$ are sixty times larger than those calculated with $\mathcal{P}_{T}(T)$. Moreover, the original term $\mathcal{P}_{T}(T)$ can stabilise the production of tryptophan $T$ by itself, exactly as we would expect from a purely proportional control. For example, it is easy to see that (2.8) is stable when we fix $E$ to be constant, that is,

$$
\frac{d T}{d t}=E^{*} \mathcal{P}_{T}(T)-\rho_{0}(T) \quad \text { with } \quad E^{*}:=k_{0} \mathcal{P}_{M}\left(T^{*}\right)
$$

Notice that $E^{*} \mathcal{P}_{T}(T)-\rho_{0}(T)$ is a strictly-decreasing function. Here the following question arises naturally: why does nature use a control system composed by the term $u=\mathcal{P}_{T}(T) E$ and the differential equation (2.9)? A first answer is the fact that the system (2.11) demands a continuous and constant production of enzyme (anthranilate synthase) $E=E^{*}$, and this production is quite expensive for the cell. However, we can deduce a second answer after comparing the whole system (2.8)-(2.9) with a (PI) control. If the term $\mathcal{P}_{T}(T)$ works as a purely proportional control, then, the inclusion of $E$ and the equation (2.9) must improve the invariance of the steady state $T^{*}$ against perturbations on the function $\rho_{0}$, just like an integral control would do. Notice that the term (2.2) is not a perfect integrator in the original system (2.1)-(2.3), for $\gamma_{E}+\mu$ is not identically equal to zero; but (2.2) is almost an integrator because the ratio $2 \frac{\gamma_{E}+\mu}{k_{E}}$ is almost zero, it is approximately equal to $6.66 \times 10^{-4}$.

We can easily check the previous hypothesis. As we know, the function $\rho_{0}$ in (2.3) and (2.8) stands for the intracellular tryptophan consumption and diffusion rates. Thus, we suppose that $\rho_{0}$ is perturbed into a new function $\rho_{\beta}$, and that this perturbation is parametrised by the parameter $\beta$. Besides, assume that $\rho_{\beta}$ is identically equal to $\rho_{0}$ whenever $\beta=0$, as well. The new steady state $T_{\beta}$ of (2.11) is the solutions of the following equation (we use $\rho_{\beta}$ instead of $\rho_{0}$ ),

$$
E^{*} \mathcal{P}_{T}\left(T_{\beta}\right)=\rho_{\beta}\left(T_{\beta}\right) \quad \text { with } \quad E^{*}:=k_{0} \mathcal{P}_{M}\left(T^{*}\right) .
$$

It is obvious that the steady state $T_{\beta}$ is a function of the parameter $\beta$ in $\rho_{\beta}$. Notice that $T_{0}=T^{*}$, because $\rho_{\beta}=\rho_{0}$ when $\beta=0$, recall equations (2.10) and (2.12). We can now calculate how large the variation of $T_{\beta}$ is with respect to the parameter $\beta$ by differentiating (2.12) with respect to $\beta$,

$$
E^{*} \mathcal{P}_{T}^{\prime}\left(T_{\beta}\right) \frac{d T_{\beta}}{d \beta}=\rho_{\beta}^{\prime}\left(T_{\beta}\right) \frac{d T_{\beta}}{d \beta}+\left.\frac{d \rho_{\beta}(s)}{d \beta}\right|_{s=T_{\beta}},
$$

and so, since $T_{0}=T^{*}$,

$$
\left.\frac{d T_{\beta}}{d \beta}\right|_{\beta=0}=\frac{1}{E^{*} \mathcal{P}_{T}^{\prime}\left(T^{*}\right)-\rho_{0}^{\prime}\left(T^{*}\right)} \times\left.\frac{d \rho_{\beta}\left(T^{*}\right)}{d \beta}\right|_{\beta=0} .
$$

Notice that both derivatives $-\rho_{0}^{\prime}$ and $\mathcal{P}_{T}^{\prime}$ in the denominator of (2.13) are negative because the functions $\rho_{0}>0$ and $\mathcal{P}_{T}>0$ are respectively strictly-increasing and decreasing. Equation 
(2.13) measures how large the variation of the steady state $T_{\beta}$ is with respect to the parameter $\beta$ in the function $\rho_{\beta}$ when the (proportional) term $\mathcal{P}_{T}(T)$ is the unique feedback control in (2.11). We must measure now how large the variation of steady state $T_{\beta}$ is with respect to $\beta$ when we use the whole feedback control $u=\mathcal{P}_{T}(T) E$ in the system (2.8)-(2.9). Rewrite (2.10) using the perturbed function $\rho_{\beta}$ instead of $\rho_{0}$,

$$
k_{0} \mathcal{P}_{M}\left(T_{\beta}\right) \mathcal{P}_{T}\left(T_{\beta}\right)=\rho_{\beta}\left(T_{\beta}\right) .
$$

Differentiating with respect to $\beta$ and rearranging terms yields :

$$
\left.\frac{d T_{\beta}}{d \beta}\right|_{\beta=0}=\frac{\left.\frac{d \rho_{\beta}\left(T^{*}\right)}{d \beta}\right|_{\beta=0}}{k_{0} \mathcal{P}_{M}^{\prime}\left(T^{*}\right) \mathcal{P}_{T}\left(T^{*}\right)+E^{*} \mathcal{P}_{T}^{\prime}\left(T^{*}\right)-\rho_{0}^{\prime}\left(T^{*}\right)} .
$$

The derivatives $\mathcal{P}_{M}^{\prime}, \mathcal{P}_{T}^{\prime}$ and $-\rho_{0}^{\prime}$ in the denominator of (2.14) are all negative, because the functions $\mathcal{P}_{M}>0, \mathcal{P}_{T}>0$ and $-\rho_{0}$ are all strictly-decreasing. We can so conclude that the absolute value of the denominator in (2.14) is larger than the absolute value of the denominator in (2.13). Therefore, using the whole feedback control $u=\mathcal{P}_{T}(T) E$ in the system (2.8)-(2.9) indeed reduces the variation $\left|\frac{d T_{\beta}}{d \beta}\right|$ of the steady state $T_{\beta}$ with respect to the parameter $\beta$ in $\rho_{\beta}$. Moreover, this variation becomes really small if the following condition holds,

$$
\left|k_{0} \mathcal{P}_{M}^{\prime}\left(T^{*}\right) \mathcal{P}_{T}\left(T^{*}\right)\right| \gg\left|k_{0} \mathcal{P}_{M}\left(T^{*}\right) \mathcal{P}_{T}^{\prime}\left(T^{*}\right)-\rho_{0}^{\prime}\left(T^{*}\right)\right|
$$

We may then safely conclude that the (proportional) term $\mathcal{P}_{T}$ strongly improves the stability of the system (2.8)-(2.9), as it was already proved in [36], while the (integral) term composed by $E$ and $\mathcal{P}_{M}$ in (2.9) indeed reduces the sensitivity of the steady state $T_{\beta}$ with respect to the variations of the parameter $\beta$ in the function $\rho_{\beta}$. This behaviour is exactly what we could expect from a Proportional-Integral control. Finally, we also have a prediction to look for in system biology. The function $\mathcal{P}_{M}$ in (2.1) and (2.9) should be steep around the steady state $T^{*}$, that is, the absolute value of the derivative $\left|\mathcal{P}_{M}^{\prime}\left(T^{*}\right)\right|$ should be as large as possible under the proper restrictions of the biological system, so that condition (2.15) holds.

\section{Positive feedback, lactose operon}

The inducible lactose (lac) operon in E. coli is the classic example of bistability. It was first noted by Monod and coworkers more than fifty years ago, although it was not fully recognised at the time. The bistable behaviour of the lactose operon has been the subject of a number of studies. It was first examined in detail by Novick and Wiener in 1957 [29] and by Cohn and Horibata in 1959 [10]. Over the last few years, the lactose operon dynamics have been analysed mathematically by several authors, the reader may find a detailed analysis in [33, 34].

Recently, Ozbudak et al. [31] performed a set of ingenious experiments that not only confirm bistability in the lactose operon when induced with the non-metabolisable inducer thio-methylgalactoside (TMG), but they also provide new and novel quantitative data that raise several questions. The action of the different feedback mechanisms can be seen in Figure 2: The enzymes $L a c Y$ 


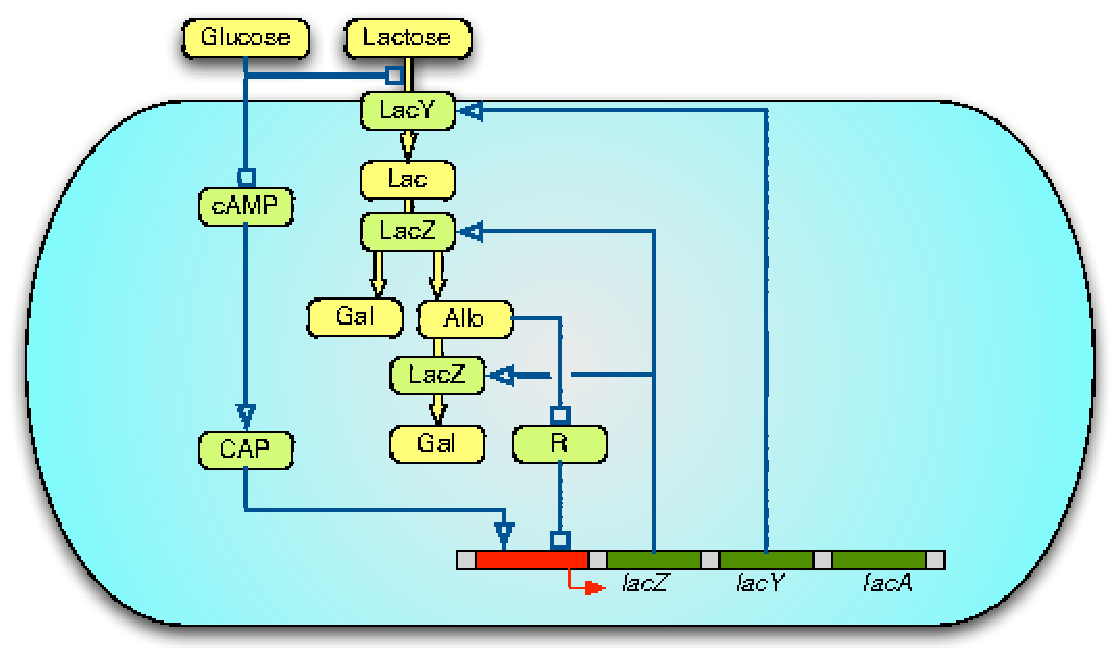

Figure 2: The lactose operon regulatory mechanisms. Figure adapted from Reference [34].

and $L a c Z$ promote their own synthesis (positive feedback in the operon) via the absorption of external lactose and its posterior transformation into galactose $(\mathrm{Gal})$ and allolactose $($ Allo); while the external glucose prevents the activation of the lactose operon via the enzymes $C A M P$ and $C A P$. Detailed model equations for the genetic regulatory system of the lactose operon have been derived in [34]. These equations can be presented as follows :

$$
\begin{aligned}
\frac{d M}{d t} & =\beta \mathcal{P}_{R}(L)-\gamma_{M} M \\
\frac{d E}{d t} & =k_{E} M-\gamma_{E} E \\
\frac{d L}{d t} & =\mathcal{P}_{L}(L) E-\gamma_{L} L .
\end{aligned}
$$

Where $M, E$ and $L$ respectively denote the mRNA, total polypeptide (lacZ), and total lactose intracellular concentration $(L a c)$. The functions $\mathcal{P}_{R}$ and $\mathcal{P}_{L}$ are smooth, non-linear, and take only positive vales. Moreover, $\mathcal{P}_{R}$ (resp. $\mathcal{P}_{L}$ ) is strictly-increasing (resp. decreasing). The factor $\beta>0$ is an external control parameter, and we have the constants :

$$
k_{E} \approx \frac{18.8}{\min }, \quad \gamma_{M} \approx \frac{0.48}{\min }, \quad \gamma_{E} \approx \frac{0.03}{\min }, \quad \gamma_{L} \approx \frac{0.02}{\min } .
$$

A complete description of the model (3.1)-(3.3) can be found in [33,34]. In particular, there are two feedback cycles competing between them, a negative one induced by the strictly-decreasing function $\mathcal{P}_{L}$, and a positive one generated by the strictly-increasing function $\mathcal{P}_{R}$. The integral character (positive or negative) of the combined cycles is then determined in (3.1)-(3.3) by the control parameter $\beta$, and obviously, by the internal structure of $\mathcal{P}_{L}$ and $\mathcal{P}_{R}$.

We assert that the system (3.1)-(3.3) works as a regenerative circuit, for there is enough evidence in [34] showing that this system has a unique stable state in the low amplification range, 
while a bistable behaviour automatically appears when high amplification rations are needed. It is well know that the positive feedback in a regenerative circuit increases its amplification ratio [3], but a really high amplification range can only be obtained when the positive feedback loop is critically tuned just below the threshold value at which the circuit would become unstable or oscillate $[4,5]$. We present in section 3.1 an introduction to the regenerative circuit in order to explain our results. Nevertheless, we need to analyse before the local stability around the steady-states $L^{*}$ of (3.1)-(3.3), which are calculated as solutions $L^{*}$ to the following equation,

$$
L^{*}=\frac{\beta k_{E} \mathcal{P}_{R}\left(L^{*}\right) \mathcal{P}_{L}\left(L^{*}\right)}{\gamma_{M} \gamma_{E} \gamma_{L}} \quad \text { with } \quad E^{*}=\frac{\beta k_{E} \mathcal{P}_{R}\left(L^{*}\right)}{\gamma_{M} \gamma_{E}}
$$

The local stability of (3.1)-(3.3) around a given steady state $L^{*}$ can be easily determined by calculating whether all the eigenvalues of the following matrix have a negative real part,

$$
A:=\left[\begin{array}{ccc}
-\gamma_{M} & 0 & \beta \mathcal{P}_{R}^{\prime}\left(L^{*}\right) \\
k_{E} & -\gamma_{E} & 0 \\
0 & \mathcal{P}_{L}\left(L^{*}\right) & -\gamma_{L}+\mathcal{P}_{L}^{\prime}\left(L^{*}\right) E^{*}
\end{array}\right] .
$$

A given steady state $L^{*}$ is then asymptotically stable whenever the three roots of the characteristic polynomial $\operatorname{det}(s I-A)$ all have a negative real part. Conversely, the steady state $L^{*}$ is unstable if at least one of the roots has a positive real part. The characteristic polynomial of $A$ can be easily calculated,

$$
\begin{aligned}
\operatorname{det}(s I-A) & =\left(s+\gamma_{M}\right)\left(s+\gamma_{E}\right)\left(s+\gamma_{3}\right)-Q_{3}, \\
\text { with: } \quad \gamma_{3} & :=\gamma_{L}-\frac{\beta k_{E}}{\gamma_{M} \gamma_{E}} \mathcal{P}_{R}\left(L^{*}\right) \mathcal{P}_{L}^{\prime}\left(L^{*}\right), \\
Q_{3} & :=\beta k_{E} \mathcal{P}_{R}^{\prime}\left(L^{*}\right) \mathcal{P}_{L}\left(L^{*}\right) .
\end{aligned}
$$

Notice that $\gamma_{3}>0$ and $Q_{3}>0$, because $\mathcal{P}_{R}, \mathcal{P}_{L}$ and $\gamma_{L}$ are all positive, and the functions $\mathcal{P}_{R}$ and $\mathcal{P}_{L}$ are respectively strictly-increasing and decreasing. Besides, $\gamma_{M}>\gamma_{E}>0$. Hence, the polynomial $\operatorname{det}(s I-A)$ is strictly-increasing for every real $s \geq 0$, and so it has a positive real root whenever $\operatorname{det}(-A)$ is negative. Conversely, we assert that the three roots of $\operatorname{det}(s I-A)$ have a negative real part if and only if $\operatorname{det}(-A)$ is positive. It follows from Strelitz's work [39] that the three roots have a negative real part if and only if the following pair of inequalities hold simultaneously:

$$
0<\gamma_{M} \gamma_{E} \gamma_{3}-Q_{3}<\left(\gamma_{M}+\gamma_{E}+\gamma_{3}\right)\left(\gamma_{M} \gamma_{E}+\gamma_{M} \gamma_{3}+\gamma_{E} \gamma_{3}\right)
$$

We have that $Q_{3}$ is positive, $\operatorname{det}(-A)=\gamma_{M} \gamma_{E} \gamma_{3}-Q_{3}$, and :

$$
\gamma_{M} \gamma_{E} \gamma_{3}<\left(\gamma_{M}+\gamma_{E}+\gamma_{3}\right)\left(\gamma_{M} \gamma_{E}+\gamma_{M} \gamma_{3}+\gamma_{E} \gamma_{3}\right)
$$

Therefore, we can easily calculate that (3.1)-(3.3) is locally stable (resp. unstable) around a given steady state $L^{*}$ whenever the following derivative is strictly less than one (resp. greater than one), recall definition (3.6),

$$
\frac{\beta k_{E}}{\gamma_{M} \gamma_{E} \gamma_{L}} \times \frac{d \mathcal{P}_{R}\left(L^{*}\right) \mathcal{P}_{L}\left(L^{*}\right)}{d L^{*}}=\frac{Q_{3}-\gamma_{M} \gamma_{E} \gamma_{3}}{\gamma_{M} \gamma_{E} \gamma_{L}}+1
$$




\subsection{The regenerative circuit}

Consider the following simplified model of a biological or electrical switch with non-linear feedback,

$$
\frac{d x}{d t}=\beta \mathcal{P}(x)-\gamma x
$$

where $\gamma>0$ is a degradation constant, $\beta>0$ is an external control parameter, and the smooth function $\mathcal{P}>0$ is non-linear. The steady states $x^{*}$ of (3.8) are the solutions to the following equation,

$$
\frac{\beta}{\gamma} \times \mathcal{P}\left(x^{*}\right)=x^{*}
$$

It is easy to prove that the dynamic system given by equation (3.8) is locally (asymptotically) stable at the steady state $x^{*}$ if and only if,

$$
\frac{\beta}{\gamma} \times\left.\frac{d \mathcal{P}(x)}{d x}\right|_{x=x^{*}}<1
$$

We choose system (3.8) because its dynamic behaviour is representative of (and similar to) the behaviour of higher dimensional systems. For example, the conditions for local stability of the steady state $L^{*}$ given in the equations (3.5) and (3.7) are completely equivalent to the pair (3.9)-(3.10), for we just need to do the following definitions,

$$
\mathcal{P}:=\mathcal{P}_{R} \mathcal{P}_{L} \quad \text { and } \quad \frac{\beta}{\gamma}:=\frac{\beta k_{E}}{\gamma_{M} \gamma_{E} \gamma_{L}}
$$

Coming back to the switch (3.8). The steady state $x^{*}$ given in (3.9) is the exit variable of the switch, while the control parameter $\beta$ allows us to choose between different values of $x^{*}$. Thus, one of the main problems is to calculate how the steady state behaves when the control parameter moves up and down. Suppose that $\beta>0$ lies in the closed interval $a \leq \beta \leq b$, and that the switch is monostable. That is, suppose that the equation (3.9) has a unique solution, and that the inequality (3.10) holds, for every parameter $a \leq \beta \leq b$. The steady state $x^{*}(\beta)$ can be then seen as a function of $\beta$; and a natural question is to determine which is the maximal possible variation of $x^{*}(\beta)$ when $\beta$ moves between the values $a$ and $b$.

Assume that the system (3.8) is also robustly stable. Notice that the model (3.8) includes both positive and negative feedback, according to the fact that $\mathcal{P}$ is either strictly-increasing or decreasing. The robust stability is granted through new safety parameters $\pi_{1} \leq \pi_{2}<1$ introduced in the equation (3.10),

$$
\pi_{1} \leq \frac{\beta}{\gamma} \times\left.\frac{d \mathcal{P}(x)}{d x}\right|_{x=x^{*}(\beta)} \leq \pi_{2}<1
$$

The parameters in (3.8) vary largely due to changes in the external variables like temperature, atmospheric pressure, $\mathrm{pH}$, salinity, et cetera. Hence, robust stability is obtained by setting the safety upper bound $\pi_{2}<1$ in (3.11); a simple estimation yields $\pi_{2} \leq \frac{3}{4}$. If the derivative $\frac{d \mathcal{P}\left(x^{*}\right)}{d x^{*}}$ is very close to $\gamma / \beta$ in (3.10), any fluctuation could make $\frac{d \mathcal{P}\left(x^{*}\right)}{d x^{*}}$ strictly greater than $\gamma / \beta$, and (3.8) will become unstable. 
Thus, suppose that (3.8) is both monostable and robustly stable for every control parameter in the closed interval $a \leq \beta \leq b$. Rewrite equation (3.9) under the assumption that the steady state $x^{*}(\beta)$ is a function of $\beta>0$,

$$
x^{*}(\beta)=\frac{\beta}{\gamma} \times \mathcal{P}\left(x^{*}(\beta)\right) .
$$

Differentiate this equation with respect to $\beta$ to give,

$$
\frac{d x^{*}(\beta)}{d \beta}=\frac{\mathcal{P}\left(x^{*}(\beta)\right)}{\gamma}+\left[\frac{\beta}{\gamma} \times\left.\frac{d \mathcal{P}(x)}{d x}\right|_{x=x^{*}(\beta)} \times \frac{d x^{*}(\beta)}{d \beta}\right]
$$

Rearranging terms and using the bounds in (3.11) yield,

$$
1-\pi_{1} \geq \frac{\mathcal{P}\left(x^{*}(\beta)\right)}{\gamma} \times\left[\frac{d x^{*}(\beta)}{d \beta}\right]^{-1} \geq 1-\pi_{2}>0 .
$$

Recall that $\mathcal{P}(\cdot) / \gamma$ is both positive and equal to $x^{*}(\beta) / \beta$. Thus, all factors in previous inequality are positive, and so we can rewrite it as follows,

$$
\frac{1}{1-\pi_{1}} \times \frac{d \beta}{\beta} \leq \frac{d x^{*}(\beta)}{x^{*}(\beta)} \leq \frac{1}{1-\pi_{2}} \times \frac{d \beta}{\beta} .
$$

Finally, integrating in the interval $a \leq \beta \leq b$, yields

$$
1 \leq\left(\frac{b}{a}\right)^{\left(1-\pi_{1}\right)^{-1}} \leq \frac{x^{*}(b)}{x^{*}(a)} \leq\left(\frac{b}{a}\right)^{\left(1-\pi_{2}\right)^{-1}} .
$$

It follows from the previous results that the hypotheses of monostability and robust stability, with a safety parameter of $\pi_{2} \leq 3 / 4$, yield an amplification exponent in (3.12) of at most $\left(1-\pi_{2}\right)^{-1} \leq 4$. This amplification exponent is quite small if we expect very large amplification, so we have to make sacrifices. Presumably, robust stability is not a condition to be sacrificed, so we have to relax the conditions over the monostability.

A solution for obtaining larger amplification exponents in (3.12) is to have a sigmoid function $\mathcal{P}(x)$. This allows the derivative $\frac{\mathcal{P}\left(x^{*}\right)}{d x^{*}}$ to be almost zero for every steady states $x^{*}$ located outside some closed interval $\left[x_{1}, x_{2}\right]$, with $x_{2}>x_{1}>0$. Conversely $\frac{d \mathcal{P}\left(x^{*}\right)}{d x^{*}}$ can be much larger than one for a third steady state $x^{*}$ inside $\left[x_{1}, x_{2}\right]$. The large value of the derivative inside the interval $\left[x_{1}, x_{2}\right]$ permits very large amplification exponents. On the other hand, even when the steady state $x^{*}$ in $\left[x_{1}, x_{2}\right]$ is unstable, the system is still stable because the large steady state $x^{*}>x_{2}$, and the small one $x^{*}<x_{1}$, are both stable. This is the solution which can be found in the genetic regulatory system of the lactose operon $[33,34]$. The Figure 3 below has a graphical representation of some steady states $x_{k}^{*}$ calculated as solutions to the equation $\gamma x^{*}=\beta \mathcal{P}\left(x^{*}\right)$ for different values of the parameter $\beta$ and two particular functions $\mathcal{P}(\cdot)$. The left picture shows the typical monostable behaviour, where the steady states are at the intersections of the graphics of $\gamma x^{*}$ (in black) and $\beta \mathcal{P}\left(x^{*}\right)$ (in vivid colours for different values of $\beta$ ). The right picture shows the typical bistable behaviour obtained after modifying the function $\mathcal{P}(\cdot)$. 

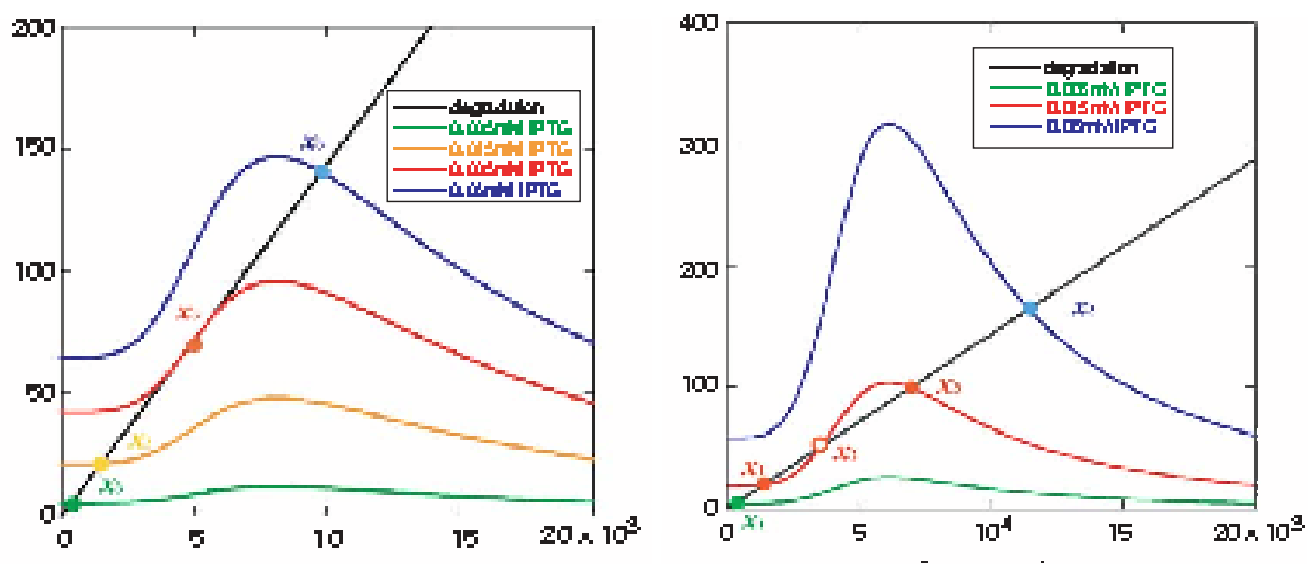

Figure 3: Monostable and Bistable Behaviour

Nevertheless, there is a second solution to the problem of obtaining larger amplification exponents in (3.12): the super-regenerative circuits. It is well know that a really high amplification range can be obtained when the positive feedback loop is critically tuned just below the threshold value at which the circuit would become unstable or oscillate $[4,5]$. In other words, we shall get infinite amplification, if we may set up the following equality,

$$
\frac{\beta}{\gamma} \times\left.\frac{d \mathcal{P}(x)}{d x}\right|_{x=x^{*}(\beta)}=1
$$

Previous condition is equivalent to set $\pi_{1}=\pi_{2}=1$ in equations (3.11) and (3.12), and so we shall obtain an infinite amplification exponent $\left(1-\pi_{1}\right)^{-1}$. However, it is impossible to set up the equality (3.13) in a real-life system, because there is always parametric variations due to external variables or thermal noise. Hence, a simpler solution is to design the feedback function $\mathcal{P}$ in such a way that the left term in (3.13) oscillates, it must be strictly grater than one half of the time, and strictly less than one the other half of the time. The switch (3.8) is then unstable half of the time, but we know exactly when it is unstable; and the condition for infinite amplification (3.13) is indeed satisfied twice every cycle. This is the basic principle of a super-regenerative circuit. We are going to extend this explanation in the next chapter. Finally, we have seen in this section that the lactose operon is a biological equivalent of the regenerative circuit, and it would be quite interesting to find an operon equivalent to the super-regenerative circuit as well, for engineering has the strange habit of imitating nature.

\section{A super-regenerative operon?}

We would want to finish this work presenting the super-regenerative circuit, which is maybe one of the simplest and most sensible detector ever invented $[4,5]$. The main idea behind this circuit is beautifully simple as well: 
Consider an electronic circuit (an oscillator) with just enough positive feedback such that it oscillates freely. Notice that the oscillations do not to start up immediately after the circuit is turned on. If the initial condition is the steady state, the circuit will stay in the steady state for ever. Nevertheless, the steady state is unstable, for there is just enough positive feedback so as to produce oscillations. Whence, any minimal perturbation (like the thermal noise) drives the circuit away from the steady state and it will then oscillate freely. Actually, we see that the oscillations grow on exponentially in magnitude until some saturation is archived. Now then, in a practical case, we see some time delay between the moment we turn on the circuit and the time when the oscillations archive their maximal magnitude.

In any case, one has to understand that any perturbation (like the thermal noise) can be the seed for the posterior oscillations in the circuit. Moreover, the stronger the seed is, the faster the oscillations archive their maximal magnitude. Well, the seed must be tuned with the natural frequency of our circuit (the oscillator), but this fact can be easily taken under consideration by designing the circuit in such a way that we known its natural oscillation frequency.

Finally, assume we have an electronic circuit as above, with just enough positive feedback such that it oscillated freely; moreover, suppose that this circuit consumes more energy when it oscillates than when it does not. We just need to turn on and off this circuit periodically (or to move the magnitude of the positive feedback up and down) in order to detect very tiny perturbations (seeds), because the bigger the seeds are, the faster the oscillations archive their maximal magnitude, and the more energy which is consume by the circuit. The consumed energy can be easily measured later.

Properly speaking, we can say that the super-regenerative circuits are so sensible that they can detect the thermal noise, because the oscillations cannot begin without thermal noise or any external perturbation (kick). It would be very interesting to find the equivalent of a super-regenerative circuit in a genetic regulatory system.

\section{Conclusions}

The idea of positive feedback has been evolving in engineering for the last one hundred years, and the idea of negative feedback is even much more older. These feedback concepts (invented or discovered by the engineers) have played a most prominent role since the first industrial revolution, and so we can expect that they also appear in the genetic regulatory systems of different operons, for the engineering has the strange habit of imitating nature. Indeed, as we said in previous sections, the tryptophan operon works as a Proportional-Integral control, and the lactose operon works as a regenerative circuit. Hence, we can find very strong tools in all the mechanical or electrical models designed by the engineers, which can be used to understand the different regulatory systems in biology. And we can also look in biology for the different models invented by the engineers, like the super-regenerative circuit. 


\section{Acknowledgements}

The author is quite grateful to the editor M. Santillán because of his opportune opinions and observations..

\section{References}

[1] H. Abraham, E. Bloch. Sur la mesure en valeur absolue des périodes des oscillations électriques de haute fréquence. Comptes Rendus de l'académie des Sciences, Vol. 168 (Juin) (1919), 1105-1108.

[2] H. Abraham, E. Bloch. Mesure en valeur absolue des périodes des oscillations électriques de haute fréquence. Annales de Physique, Vol. 12 (1919), 237-252. Reprinted in: J. Phys. Theor. Appl. Vol. 9 (1919), 211-222.

[3] E.H. Armstrong. Wireless receiving system. U.S. Patent 1,113,149. Filed on October 29, 1913. Issued on October 6, 1914.

[4] E.H. Armstrong. Signalling system. U.S. Patent 1,424,065. Filed on June 27, 1921. Issued on July $25,1922$.

[5] E.H. Armstrong. Some recent developments of regenerative circuits. Proceedings of Institute of Radio Engineering, Vol. 10 (1922), No. 4, 244-260.

[6] H.S. Black. Wave translation system. U.S. Patent 2,102,671. Filed on April 22, 1932. Issued on December 21, 1937.

[7] A. Callender, D.R. Hartree, A. Porter. Time-lag in a control system. Philosophical Transactions of the Royal Society of London, Series A, Vol. 235(756) (1936), 415-444.

[8] W.B. Cannon. The wisdom of the body. W. W. Norton, New York, 1932.

[9] J.D. Chung, G. Stephanopoulos. On physiological multiplicity and population heterogeneity of biological systems. Chem. Eng. Sci., Vol. 51 (1996), 1509-1521.

[10] M. Cohn, K. Horibata. Analysis of the differentiation and of the heterogeneity within a population of Escherichia coli undergoing induced beta-galactosidase synthesis. J. Bacteriol., Vol. 78 (1959), 613-623.

[11] E.H. Colpitts. Oscillation generator. Canadian Patent 203,986. Filed on January 20, 1919. Issued on September 14, 1920.

[12] E.B. Craft, E.H. Colpitts. Radio telephony. Tran. A.I.E.E., Vol. 38 (Feb.) (1919), 305-343.

[13] R. Dennhardt. Die Flipflop-Legende un das Digitale. Doctor Philosophiae Dissertation, Humboldt Universität, Berlin, 2003. 
[14] W.H. Eccles, F.W. Jordan. A Method of using two triode valves in parallel for generating oscillations. The Electrician, Vol. 83 (Sep. 19) (1919), 299.

[15] W.H. Eccles, F.W.Jordan. A Trigger relay utilising three-electrode thermionic vacuum tubes. The Electrician, Vol. 83 (Sep. 19) (1919), 298.

[16] W.H. Eccles, F.W.Jordan. A trigger relay utilising three-electrode thermionic vacuum tubes. Radio Review, Vol. 1, No. 3 (Dec.) (1919), 143-146.

[17] M. Freeman. Feedback control of intercellular signalling in development. Nature, Vol. 408 (Nov. 16) (2000), 313-319.

[18] H. Garnsback. The crystodyne principle. Radio News, Vol. 431 (1924), 294-295.

[19] R. Hartley. Oscillation generator. U.S. Patent 1,356,763. Filed on June 1, 1915. Issued on October 26, 1920.

[20] D.R. Hartree, A. Porter, A. Callender, A.B. Stevenson. Time-lag in a control system. II. Proceedings of the Royal Society of London, Series A, Vol. 161 (907) (1937), 460-476.

[21] H.L. Hazen. Theory of servomechanisms. J. Franklin Institute, Vol. 218, No. 3 (Sep.) (1934), 279-331.

[22] A. Ivanoff. Theoretical foundations of the automatic regulation of temperature. J. Institute Fuel, Vol. 7 (1934), No. 33. 117-138.

[23] J. Keener, J. Sneyd. Mathematical Physiology. Springer-Verlag, New York, 1988.

[24] L. Maloney, B. Rotman. Distribution of suboptimally induces -D-galactosidase in Escherichia coli. The enzyme content of individual cells. J. Mol. 1973, Vol. 73 (1973), 77-91.

[25] A. Marinčić. Nikola Tesla Colorado Springs Notes, 1899-1900. Nolit, Beograde, 1978. www.bibliotecapleyades.net/tesla/coloradonotes/coloradonotes.htm

[26] J.C. Maxwell. On governors. Proc. R. Soc. lond., Vol. 16 (1867-1868), 270-283.

[27] W.S. McCulloch, W. Pitts. A logical calculus of the ideas immanent in nervous activity. Bulletin of Mathematical Biophysics, Vol. 5 (1943), 115-133. Reprinted in: Bulletin of Mathematical Biology, Vol. 52 (1990), No. 1-2, 99-115.

[28] N. Minorski. Directional stability of automatic steered bodies. J. Am. Soc. Naval Engrs., Vol. 34 (1922), No. 2, 280-309.

[29] A. Novick, M. Wiener. Enzyme induction as an all-or-none phenomenon. Proc. Natl. Acad. Sci. USA, Vol. 43 (1957), 553-566.

[30] Ş. Odobleja. Psychologie consonantiste, vol. I et II. Librairie Maloine, Paris, 1938-1939. 
[31] E.M. Ozbudak, M. Thattai, H.N. Lim, B.I. Shraiman, A. van Oudenaarden. Multistability in the lactose utilization network of Escherichia coli. Nature, Vol. 427 (2004), 737-740.

[32] A. Rosenblueth, N. Wiener, J. Bigelow. Behavior, Purpose and Teleology. Philosophy of Science, Vol. 10 (1943), No. 1. 18-24.

[33] M. Santillán, M.C. Mackey. Influence of catabolite repression and inducer exclusion on the bistable behavior of the lac operon. Biophys. J., Vol. 86 (2004), 1282-1292.

[34] M. Santillán, M.C. Mackey, E.S. Zeron. Origin of bistability in the lac operon. Biophys. J., Vol. 92 (June) (2007), 3830-3842.

[35] M. Santillán, E.S. Zeron. Dynamic influence of feedback enzyme inhibition and transcription attenuation on the tryptophan operon response to nutritional shifts. J. Theor. Biol., Vol. 231 (2004), 287-298.

[36] M. Santillán, E.S. Zeron. Analytical study of the multiplicity of regulatory mechanisms in the tryptophan operon. Bull. Math. Biol., Vol. 68 (2006), No. 2, 343-359.

[37] O.H. Schmitt. A thermionic trigger. J. Sci. Instrum., Vol. 15 (1938), 24-26.

[38] A.I. Shumilinal, V.A. Makarov. Milestones in P. K. Anokhin's scientific career (on the 90th anniversary of his birth). Neuroscience and Behavioral Physiology, Vol. 18 (1988), No. 4, 269-274.

[39] S. Strelitz. On the Routh-Hurwitz problem. Am. Math. Mon., Vol. 84 (1977), No. 7, 542-544.

[40] L.B. Turner. The Kallirotron. An aperiodic negative-resitance triode combination. Radio Review, Vol. 1 (April) (1920), 317-329.

[41] L.B. Turner. The Kallirotron. To the Editor of the Radio Review. Radio Review, Vol. 1 (1920), 523.

[42] N. Wiener. Cybernetics or control and communication in the animal and the machine. MIT Press, Cambridge, 1964. 